\section{Una experiencia desconocida: las Misiones Socio-Pedagógicas en Uruguay (1945-1959)}

Gabriel Scagliola

\section{Resumen}

En el año 1945, surgen en Uruguay las Misiones Socio-Pedagógicas, que fueron organizadas por estudiantes de magisterio a los que se irán sumando los de medicina, de odontología, de agronomía, de la universidad del trabajo; procurando acercar prácticas de higiene, de salud, de vivienda allí donde la pobreza daba cuenta del abandono en que transcurría la vida de la inmensa mayoría de la población rural. Además, llevaban cine, títeres, teatro, juegos, baile y música a la escuela rural.

Durante décadas, fueron olvidadas; sin embargo, el registro de ese trabajo existe disperso en archivos particulares y publicaciones diversas y en la memoria de los hombres y mujeres que participaron. Este trabajo es uno más de esos esfuerzos que se están llevando a cabo para recuperar su memoria.

\section{An unknown experience: Socio-Pedagogical Missions in Uruguay (1945-1959)}

\begin{abstract}
In the year 1945, the Socio-Pedagogical Missions emerged in Uruguay, which were organized by teaching students to which will be added those of medicine, of dentistry, of agronomy, of the university of work; trying to bring hygiene practices, health, housing where poverty accounted for the abandonment in which passed the lives of the vast majority of the rural population. They also brought movies, puppets, theater, games, dance and music to the rural school.
\end{abstract}

For decades, they were forgotten; however, the record of that work exists scattered in particular archives and diverse publications and in the memory of the men and women

\section{Palabras clave:}

Misión Socio-Pedagógica, maestro, universidad popular, estudiantes, congreso, escuela rural.

\section{Keywords:}

Socio-Pedagogical Mission, teacher, popular university, student, congress, rural school. 
who participated. This work is one more of those efforts that are being carried out to recover his memory.

\section{Introducción}

En la primera mitad del siglo XX, comenzaron a tomar forma desde el movimiento estudiantil latinoamericano las primeras propuestas de vinculación de los institutos de educación superior con la sociedad. El eje articulador de estos planteos fueron los congresos estudiantiles que permitieron el debate de ideas tales como la extensión, universidades populares, difusión y el acceso a la cultura en general (Palacios, 1908).

El Primer Congreso Internacional de Estudiantes Americanos, reunido en Montevideo entre el 26 de enero y el 2 de febrero de 1908, propuso la instrumentación de programas dirigidos a "difundir la cultura intelectual en la sociedad" (Evolución, 1912, p.33). Dos años después, en Buenos Aires, el Segundo Congreso Internacional de Estudiantes Americanos resolvía recomendar la extensión universitaria como el medio para obtener "la elevación intelectual y moral del pueblo", y establecía que en los lugares donde no hubiere universidades, los estudiantes debían ayudar a la fundación "de salones o clubes obreros" y realizar "cátedras populares y conferencias públicas" (Evolución, 1912, p.88). A partir del Primer Congreso Nacional de Estudiantes Universitarios, organizado por la Federación Universitaria Argentina en 1918, la extensión universitaria estará en "programas de los centros y federaciones estudiantiles de toda nuestra América" (Del Mazo, 1946, citado por Bralich, 1986, p.22). En 1921, el Primer Congreso Internacional de Estudiantes reunido en México, aprobó como resolución:

Que la extensión universitaria es una obligación de las asociaciones estudiantiles, puesto que la primera y fundamental acción que el estudiante debe desarrollar en la sociedad es difundir la cultura que de ella ha recibido entre quienes la han menester.

Asimismo, manifestaba:

[...] Es una obligación de los estudiantes el establecimiento de universidades populares, que estén libres de todo espíritu dogmático y partidista y que intervengan en los conflictos obreros inspirando su acción en los modernos postulados de justicia social. (Del Mazo, 1941, p.86-88)

En el mismo sentido, los estudiantes magisteriales desarrollaron paralelamente otras vías de concreción y relacionamiento con la sociedad, a partir de las experiencias de las Misiones Culturales mexicanas y Pedagógicas españolas. Las Misiones SocioPedagógicas surgidas en el año 1945, como preparación para quienes optarían por la escuela rural y como forma de conocer y denunciar la situación del Uruguay rural (Bralich, 1986). En 1949 se integraron en forma masiva estudiantes universitarios y los institutos normales de los diferentes departamentos empezaron a organizar sus propios Centros de Misiones. Los Centros de Misiones Socio-Pedagógicas eran las instituciones encargadas de la coordinación del trabajo en las misiones entre los grupos:

En algunos casos, muy al comienzo de la tarea, se fundó el Centro, en otros se fue creando con la evolución del trabajo, ya muy avanzado este. Lo que nos interesa destacar es el surgimiento de nuevos centros por todo el país y la vinculación que estos mantienen por correspondencia en algunas oportunidades; en algunos casos observadores misioneros de un centro son invitados a concurrir a la misión que organiza otro. (Silveira, 1960) 
Nuestro objeto de estudio son las Misiones Socio-Pedagógicas. Aunque estas misiones se extendieron hasta el año 1971, este trabajo se centrará en las primeras Misiones Socio-Pedagógicas, entre la primera misión en 1945 y la creación del Departamento de Extensión y Acción Social en la Universidad de la República en 1959.

\section{Corrientes de pensamiento y acción que van a confluir en el movimiento misionero}

En el surgimiento de las Misiones Socio-Pedagógicas podemos identificar la síntesis de al menos dos tradiciones:

\section{a. Normalista}

Durante la dirección de María Orticochea en los Institutos Normales de Montevideo, se producirán los primeros ensayos de misiones pedagógicas, organizados para conocer el medio donde irían a trabajar y que les era desconocido. La primera crónica de estas misiones pertenece al año 1941, con una visita a la escuela n. 56 de Estación González, departamento de San José (Clemente Batalla, 2008, p.32), donde "las alumnas normalistas tuvieron a su cargo diversas lecciones muy interesantes, así como también Dirección de Coros e interpretaciones al piano; el profesor Zarrilli dirigió las dramatizaciones" (Orticochea, 1941, p.86).

Asimismo, la Inspectora de Escuelas de Práctica Blanca Samonatti de Parodi, ${ }^{1}$ presenta al Consejo Nacional de Enseñanza Primaria y Normal el proyecto para realizar misiones pedagógicas. En la fundamentación define a la Misión Pedagógica como una "Escuela Normal Volante" (Samonatti, 1946, p.182), con dos fines: uno de superación docente y otro de ampliación cultural. Se constituiría con personal del Departamento de Práctica: un inspector (que sería el director de la Misión), algunos directores, ayudantes, un profesor de Pedagogía de los Institutos Normales, un profesor de Música, un profesor de Plástica, el Jefe de Cinematografía Escolar, un especialista en el estudio de la naturaleza, un profesor de alimentación racional y una visitadora social.

Las actividades programadas eran: dar a conocer material didáctico, conferencias sobre temas de la Escuela Nueva, lecciones de práctica docente, conversaciones con proyecciones, cursos cortos, asociación del arte a la enseñanza, higiene, alimentación, etcétera, así como también la realización de espectáculos musicales y lecturas comentadas (Samonatti, 1946, p.183).

En este contexto, y con una clara voluntad de estimular a los alumnos normalistas a la realización de esta tarea, se dedicó el Concurso Anual de Pedagogía para estudiantes a las Misiones Pedagógicas. La entonces estudiante, Amelia Esteva, obtiene el segundo premio. Su artículo sería publicado un año después.

En su propuesta planteaba la posibilidad de realizar las misiones pedagógicas en las vacaciones o al inicio del año escolar y la duración debía de ser "variable según las circunstancias” (Esteva, 1948, p.485). Estarían integradas por maestros asesorados "por elementos preparados de las facultades" (Esteva, 1948, p.476). Los aspirantes seleccionados deberían realizar un curso preliminar ${ }^{2}$ que les servirá para renovar y ampliar conocimientos y ponerlos en contacto con la situación del país.

En el curso de perfeccionamiento se establecían una serie de materias:

»Filosofía de la Educación
1. Nació el 3 de julio de 1892 en La Paz, Departamento de Colonia, y murió el 16 de noviembre de 1964 en Montevideo. "Recibida de Maestra de Primer Grado, continuó estudiando y obtuvo el título de Segundo; pero como existía otro título, aún superior, el de Tercer Grado, hasta él llegó. Fueron pocos los Maestros que lo obtuvieron. [...] En los Institutos Normales fue Profesora de Pedagogía de los aspirantes al título de Segundo Grado. [...] Preside por muchos años, una de las Comisiones que recorre varios departamentos tomando exámenes de aspirantes a Maestros...". Ocupó diversos cargos en Primaria y en los Normales, fue inspectora de Escuelas de Práctica. Años después, coronando su carrera, la designan, por dos períodos, desde 1952 a 1959, vocal del Consejo Nacional de E. Primaria y Normal. "Suyo es el Proyecto de Reforma del Plan de Estudios Magisteriales, suprimiendo el Ciclo Cultural de cuatro años, equivalente a los estudios de Secundaria; ingreso con el ciclo Liceal aprobado, implantación del año Preparatorio y prolongación del Ciclo Profesional en un año más" (D’Auria, 1964, p.123-124).

2. En México la realización de cursos de preparación era una práctica habitual. Según don Lucas Ortiz Benítez, primer director del CREFAL, en 1926 se realizaron los primeros cursos de perfeccionamiento especiales para misioneros. Para cada grupo había clases determinadas. Al concluir los cursos los misioneros recibían un pliego de instrucciones, entre las cuales, había un párrafo destinado a señalar cómo emplear la biblioteca ambulante y los equipos de carpintería e industrias (Ortiz Benítez ,1952). 
3. La directora de los Institutos Normales, María Orticochea, antes de la realización de las Misiones reunía a las integrantes y las responsabilizaba de la moral, según las entrevistas realizadas a Dana Sosa y Gladys Méndez (1987).

4. La Exposición de Material didáctico de las Escuelas de Práctica fue realizada en el Museo Pedagógico, desde el 19 de octubre hasta el 11 de noviembre de 1944. La inspectora de Escuelas de Práctica prof. Blanca Samonatti de Parodi expresa: “... no son las escuelas de nuestra Capital las que más necesitan de sugestiones y de modelos imitativos; sino las de los departamentos del interior. Con el deseo de llegar hasta ellas en la realidad de los hechos, [...] elevé a la consideración de la Superioridad otro proyecto; el de una Misión Pedagógica que, entre sus otros cometidos, tuviera el de ofrecer a la divulgación las piezas que, por su naturaleza, fuera posible imitar en cualquier parte. Este carácter 'rodante' es el que asignará el material, su verdadero propósito inicial". "Muestra Didácticas de las Escuelas de Práctica de la Capital”. (Anales de Instrucción Primaria,1946, p.256).
" Estudio de la vida social y rural

"Higiene

» Puericultura y Alimentación Infantil.

$»$ Cocina

" Agricultura

» Organizaciones deportivas, de festivales y escenografía

Adicionalmente en las de Higiene, Agricultura, Cocina se contaría con personal especializado: medico higienista, enfermera, visitadora social y agrónomo.

Asimismo, valora el éxito de la misión no solo en la buena organización y en la selección de las personas, sino también en "el gran valor moral de los que la integran" (Esteva, $1984, \mathrm{p} .477) .^{3}$

La misión pedagógica se dividiría en tres tipos de actuación: Cultural, Social y de Superación Docente.

Se establecería la acción cultural, mediante la creación de bibliotecas populares fijas y circulantes, las lecturas públicas y conferencias sobre temas culturales, cine, teatro, coros, pequeñas orquestas y títeres y las exposiciones reducidas de obras de arte. Además de cuadros, esculturas y trabajos manuales, menciona la posibilidad de incorporar la muestra didáctica, que en esos momentos se exponía en el Museo Pedagógico, donde se da a conocer "un extraordinario material de enseñanza y juegos educativos, que se usan en la capital" (Esteva, 1984, p.480). ${ }^{4}$

Entendía que la misión pedagógica con este fin único, se desarrollaría en los núcleos urbanos, ciudades o pueblos de alguna importancia. “¿Pero, los demás? A los desheredados, ¿qué se les daría? Una palabra vana, donde las actuaciones puramente espirituales, serian semillas caídas en suelo estéril y no fructificaran. De ahí que sea necesaria, imprescindible, una actuación social inmediata y eficaz" (Esteva, 1948, p.480).

En tanto que para la actuación social de la Misión entendía necesario dotar a la escuela a ser misionada de material útil, comedor escolar, ropero escolar, charlas de divulgación de nociones higiénicas, actuación médica directa y huerto escolar.

Por último, para la Superación Docente de la Misión proponía:

... Cursos para maestros sobre temas tales como: el examen de la realidad natural y social que rodea la escuela tratando de mostrarles el modo de utilizarla con fines educativos, excursiones conjuntas de maestros y niños, lecciones prácticas de literatura y lenguaje, lecciones de ciencias con aplicación al medio rural, conversaciones sobre asuntos de la Escuela Nueva, demostraciones de técnicas docentes y la asociación del arte con la enseñanza, realización personal de trabajos como complemento de los temas teóricos y el estudio de la forma de aprovechar el juego como uno de los recursos más poderosos que intervienen en la educación. (Esteva, 1948, p.484)

Eran cursos muy similares a los realizados por las misiones pedagógicas de la República Española donde celebraban una semana o quincena pedagógica en una escuela, para que concurrieran los maestros a un curso de perfeccionamiento.

\section{b. Las universidades populares}

Las universidades populares surgen en Francia, España y Alemania a finales del siglo XIX y principios del XX, esparciéndose por diversos países de Latinoamérica. En Uruguay, la Reforma Universitaria de Córdoba repercutió en el movimiento estudiantil 
y los postulados de la Reforma fueron incorporados a los reclamos, pero "la aplicación no podía ser mecánica" ya que "muchos de sus puntos no vinieron sino a encuadrarse en la tradición liberal que caracterizaba a nuestra Universidad desde el siglo anterior...". Además, algunos de sus postulados, ya se habían concretado: la Ley Orgánica de 1908 incorporaba la representación indirecta de los estudiantes en los Consejos Directivos; en 1914 el ex dirigente estudiantil y entonces Ministro de Instrucción Pública y Baltasar Brum había presentado al Parlamento un proyecto de cátedras libres; y en 1916, el Parlamento aprobaba la ley que exoneraba de matrícula y examen a los estudiantes de enseñanza secundaria.

En 1917, se produjeron dos acontecimientos de importancia para el movimiento estudiantil: La disolución de la Federación de Estudiantes del Uruguay $(\mathrm{FEU})^{5}$ que condujo a la desunión y al aislamiento de los centros estudiantiles y la fundación del Centro de Estudiantes Ariel. El nuevo Centro congregó a quienes tenían "una gran inquietud intelectual" y estaban interesados por los "problemas sociales y educativos del país" pero no representaba a ninguna facultad ni participaba como "entidad coordinadora central" de los universitarios (Van Aken, 1990).

La propuesta de creación de las Universidades Populares fue planteada en el Congreso Nacional de Estudiantes de 1930, por el bachiller Héctor González Areosa, en representación del Centro de Estudiantes Ariel. El informe, que acompañaba la iniciativa, denunciaba la concentración de la propiedad de la tierra en manos de los grandes latifundistas ${ }^{6}$ y establecía la necesidad de crear Universidades Populares con el apoyo de estudiantes, profesionales, intelectuales y dirigentes sindicales; con el objetivo de educar a las clases trabajadoras urbanas y rurales, y así hacerlas conscientes de su condición de explotadas.

Entre todas, la más prestigiosa, fue la Universidad Popular Central. Fundada, en 1938, con representantes de diversas instituciones: Ateneo de Montevideo, Federación de Estudiantes Universitarios del Uruguay (FEUU), ${ }^{7}$ Unión Nacional del Magisterio, Agrupación de Intelectuales, Artistas, Periodistas y Escritores (AIAPE) y Escuela Taller de Artes Plásticas (ETAP) convocando:

Ha surgido la iniciativa, ya en andamiento, de fundar una gran entidad con la denominación de UNIVERSIDAD POPULAR CENTRAL. Para la plena realización de este propósito, que concita nuestros mejores afanes, es preciso agrupar en torno de la nueva institución a los ciudadanos que en el momento histórico actual afirman con dignidad y resolución su fe en los auténticos principios democráticos que postulan una constante y libre actividad cultural en el seno del pueblo. (Acción, 1938, p.2)

Los cursos eran gratuitos y se confeccionaban según solicitudes, propuestas y sugerencias de los propios estudiantes. Comprendían desde alfabetización, pasando por los de capacitación técnica, hasta los cursos superiores o de ampliación cultural: Analfabetos y Semianalfabetos; Aritmética, Ortografía y Redacción; Gramática, Corte y Confección, Ciencias Geográficas, Francés, Inglés, Contabilidad, Taquigrafía, Dibujo Lineal, Dibujo Artístico, Castellano para Extranjeros, Ingreso a Secundaria, Solfeo, Encuadernación, Economía Política, Anatomía, Fisiología e Higiene.

La creación de la Sección Interior en la Universidad Popular Central va a tener amplia repercusión. Formada, fundamentalmente, por integrantes de la Unión del Magisterio tenía como principal objetivo la difusión de la cultura en toda la República, propiciando relaciones de intercambio y ayuda mutua con diversas instituciones culturales y deportivas como "Renovación" de Florida, "Cultura y de Deporte" de Melo, "José Enrique Rodó" de Juan L. Lacaze, "Elevación” de Dolores, "Escuela Experimental” de Progreso, "Gabriela Mistral” de Canelones y "Florencio Sánchez" de Dolores (AIAPE, 1939, p.12).
5. Fundada en 1909 , fue conocida como la "FEU de 'La Cumparsita", debido a que el famoso tango de Mattos Rodríguez fue compuesto en el local de la Asociación de Estudiantes de Medicina (Van Aken, 1990).

6. Poco tiempo después, lo mismo hacían los maestros: "El so\% de los propietarios posea el $5 \%$ de la propiedad territorial rural; el $5 \%$ posea el $50 \%$ de la tierra y el restante $45 \%$ posea el $45 \%$ de la propiedad rural. [...] La presencia de los rancheros, forma extrema del minifundio. [...] Julio Castro estimaba su número en 587 , con aprox. 120.000 habitantes, entre ellos de 20.000 a 25.000 niños en edad escolar, personas, dice Castro, "que viven por debajo de lo que podrá considerarse nivel de vida humano"” (Soler Roca, 2011). 7. Fundada el 26 de abril de 1929. Estuvo integrada en sus inicios por la Asociación de Estudiantes de Medicina, Asociación Uruguaya de Estudiantes de Notariado, Asociación de Estudiantes de Agronomía, Centro de Estudiantes de Derecho, Asociación Estudiantes de Farmacia, Centro de Estudiantes de Arquitectura, Centro de Estudiantes de Odontología, Asociación de Estudiantes de Veterinaria, Centro de Estudiantes de Ingeniería y Centro Cultural Liceo Nocturno (Sanguiñedo, 2014). 
8. “ [Un] Conjunto de viviendas de la gente que ya no tiene lugar determinado en la vida social y económica y se ha ido a establecer en esos lugares. No sabemos aún que factores determinan la selección del lugar para este agrupamiento humano. Quizás la equidistancia de sus primitivos sitios de residencia. La existencia de leña y agua, la disponibilidad de tierras fiscales o 'tierras de nadie' o la proximidad a la estancia o al caserío, que puedan ofrecer oportunidad de trabajo para hombres y mujeres. El hecho es que en la vasta campiña uruguaya, agrícola o ganadera existe el rancherío. [...] Albergan a más de cien mil personas, las que están viviendo en las más terribles condiciones humanas, las que han llevado a desmejorar con el agraviante nombre de 'pueblos ratas'”. (Castro, 1985, p.22).

9. Entre los más destacados: Julio Castro, Ana María Clulow y Jesualdo.
Entre las actividades realizadas se destacaban:

a) Donaciones de libros, destinadas a aumentar los fondos de las Universidades y Bibliotecas Populares del interior.

b) Ciclo de Conferencias, organizada junto a la Comisión Cultura, a cargo de distintos periodistas y escritores. En el Primer Ciclo intervinieron los escritores Justino Zavala Muniz, Serafín J. García, Juan José Morosoli, Santiago Dossetti, Francisco Espínola y Alfredo Lepro. Todos coincidían en el diagnóstico: el latifundio, el minifundio, el analfabetismo, las enfermedades y vicios sociales, eran resultado de la falta de trabajo y de la pobreza en el medio rural. Asimismo, reclamaban la creación de Universidades Populares "apartadas de los rigorismos oficiales, de las retóricas, de las necesidades utilitarias y, naciendo en el seno del pueblo, del pueblo y para el pueblo" (Zavala Muniz, 1940, p.18).

En 1941, Danilo Trelles realizaba una evaluación del trabajo realizado por las Universidades Populares en el campo:
... Allí no hay medios con que lograr una cultura. Que vaya alguno a convencerlos de que es necesario instruirse, y se le reirán en la cara. Y tendrán toda la razón del mundo. Cuando urgencias económicas torturan el estómago, no hay tiempo para pensar en eso. Podría hacerlo el hijo del estanciero o del chacrero rico, cuyo esfuerzo no fecunda la simiente, cuyos dedos no desmenuzan los terrones, y que tiene el espíritu tan lejos de todo esto. (Trelles, 1941, p.12)

Coincidente con la valoración que Julio Castro realizará de experiencias anteriores durante la primera Misión Socio-Pedagógica al rancherío ${ }^{8}$ de Caraguatá: “... con cultura solo en campaña, no hacemos nada" (Castro, 1985, p.22).

La Universidad Popular Central se mantuvo hasta el año 1942 cuando se "disolvió por cesación de pagos" (Van Aken, 1990). Desbaratada la experiencia, algunos integrantes de la Sección Interior se integran a los Institutos Normales como profesores. ${ }^{9}$

\section{Las Misiones Socio-Pedagógicas}

\section{Los modelos}

Los dos más cercanos antecedentes de las Misiones Socio-Pedagógicas uruguayas se desarrollaron por iniciativas estatales en contextos de profundos cambios políticos: en el México revolucionario de los años veinte y en la España republicana de los años treinta.

Fue en estos dos países donde la palabra misión, que hasta el momento había estado asociada a la evangelización, empezó a emplearse para denominar a un cierto tipo de intervención sociocultural laica que intentaba paliar la desigualdad manifiesta entre las posibilidades educativas que podían hallarse en la ciudad y en el campo. Ambos modelos -el cultural mexicano y el pedagógico español- optarían por hacerlo de diferente modo, aunque sus intenciones iban encaminadas al mismo fin: extender los derechos de la ciudadanía - a la comida, a la educación, al trabajo o la cultura- a aquellos que ni siquiera sospechaban que existían estos derechos.

En 1923, se crearon las misiones culturales mexicanas. La revolución, que en las zonas rurales se había hecho al grito de “iTierra y escuelas!”, no sabía cómo educar a una población con gran diversidad étnica y lingüística, sin maestros preparados ni planes de estudios adecuados (Secretaría de Educación Pública, 1928). Las misiones pedagógicas españolas, nacidas en 1931 casi a la par que la Segunda República, decidieron 
enfrentarse a distintos problemas. Dejando para el Estado la lucha contra el analfabetismo y el fortalecimiento de las escuelas, estas misiones reclamaron para sí la tarea de "llevar a las gentes, con preferencia a las que habitan en localidades rurales, el aliento del progreso y los medios de participar en él [...] de modo que los pueblos todos de España, aun los apartados, participen en las ventajas y goces nobles reservados hoy a los centros urbanos" (García Alonso, 2003). Educar a través de la música, el cine, el teatro; crear museos en cuadras y pajares; recitar romances en las plazas; incitar la risa con los títeres. Sin embargo, la influencia que este planteamiento haya podido tener en la experiencia uruguaya fue recibida a través de la misión pedagógico-social de Sanabria, dirigida por Alejandro Casona, en la que se intentó consensuar este estilo de "farándula justiciera" con la mejora material de las condiciones de vida de la población. La guerra civil acabó con este proyecto democrático y los misioneros pagaron con la muerte, la cárcel o el exilio haber defendido por las aldeas de España el derecho de los pobres a soñar.

\section{La primera misión}

En el año 1945, el estudiante normalista Moisés Lasca era presidente de la Asociación de Estudiantes Magisteriales desde donde:

\begin{abstract}
... La Directiva de Estudiantes de Magisterio (primer grado) empezó a organizar desde principios de año lo que fue la primera misión. [...] Fuimos separando de la Asociación el núcleo de gente que trabajaba, para independizarlo de todos los problemas y dedicarlo exclusivamente a la misión; entre los estudiantes que mostraban más interés, más capacidad. (Gándaro, s./f.)
\end{abstract}

Ese grupo quedaría conformado por María C. Mercader, Sofia Buzó, Aida Caro Betelú, Marta Laporta, Elsa R. Dollenarte, Fanny D. Bonino, Alcira Cardozo, Rubén García, Estanislao O. Acosta, Osvaldo González, Jaime López, Cesar Vallejo, Rubén Fernández Chaves, Rubén Benítez, José P. Nacimiento y Moisés Lasca. Asimismo, se cursaron invitaciones a la Asociación de Estudiantes de Medicina y al Centro de Estudiantes de Derecho para que enviaran delegados. Juan Gómez Gotuzzo fue el representante de la Asociación de Estudiantes de Medicina ("Una sombría realidad social fue observada por la misión pedagógica en los rancheríos de Caraguatá", 15 de julio 1945, El Día, p. 7). ${ }^{10}$ Pero el grupo no estaría completo. La directora de los Institutos Normales, María Orticochea, exigiría la presencia de profesores acompañantes: Francisco "Pancho" Olivares, Josefa Arrien Jaureguiberry y Julio Castro ${ }^{11}$ (Castro, 1985). Completaría el grupo el operador cinematográfico Atilio Saturno.

El lugar a misionar, Caraguatá, ${ }^{12}$ ubicado en la 8.a Sección del Departamento de Tacuarembó, fue elegido por sugerencia del director del Consejo de Enseñanza Primaria y Normal, Arquitecto Pérez Montero, quien "ayudó con una fuerte suma de dinero y los pasajes" (Castro, 1985, p.22). Recibieron, además, el aporte de trescientos pesos de la Asociación de Amigos de los Institutos Normales, mientras el Ministro de Instrucción Pública "ni siquiera se dignó recibirlos, tal vez porque no eran gentes de cuello duro" (Castro, 1985, p.22).

La misión, a desarrollarse entre los días 2 y 11 de julio, ${ }^{13}$ tendría como finalidad:

1. Llevar al lugar que se visite todo lo que pueda contribuir al mejoramiento físico y espiritual de sus habitantes. 2. Que los integrantes de la misión tengan una experiencia viva de la campaña, de sus problemas y formas de solucionarlos o atenuarlos. 3. Elevar un informe a las autoridades competentes, de las necesidades de la zona, para que traten de mejorarla". (Inquietud, órgano estudiantil de los Institutos Normales, junio 18 de 1945, citado por Bralich, 1986, p. 19-20)
10. El profesor Jorge Bralich establece "unos veinte misioneros entre los cuales había estudiantes de magisterio, de medicina, y algunos maestros" (Bralich, 1986). El doctor Felipe Cantera afirma que "el grupo estaba integrado veinticinco estudiantes y dos profesores, Julio Castro y Josefa Arrién" (Cantera, 1968). El maestro Julio Castro plantea la siguiente integración: "dieciocho estudiantes de magisterio -varones y muchachasy tres profesores: la señorita Josefa Arrién, el señor Pancho Oliveras y el que esto suscribe" (Castro, 1985). 11. Julio Castro era docente de Filosofía de la Educación en los Institutos Normales y había sido maestro de Moisés Lasca en la

Escuela n.․9 93. Josefa Arrién era maestra jubilada y tía de Marta Mercader. 12. La maestra Elsa Fernández y el doctor Borges ya venían trabajando junto con el director del Consejo de Educación Primaria y Normal en la elaboración del Proyecto de Centros de Recuperación a instalar en la 8. a Sección del Departamento de Tacuarembó. 13. Generalmente se ha pensado que la Misión Pedagógica a Caraguatá fue realizada entre el 4 y el 19 de julio de 1945. Pero: El País establece como fecha de partida el 2 de julio de 1945. El Día del jueves 12 que anunciaba la llegada. "En las últimas horas de anoche arribó a la Estación Central la Misión Pedagógica que integrada por estudiantes de $6 .^{\circ}$ y $7^{\circ}$ años de los Institutos Normales actuará en el núcleo escolar de Caraguatá en el de partamento de Tacuarembó" ("Llego de Caraguatá la misión pedagógica escolar", 12 de julio de 1945, El Día, p.8). 
Julio Castro reseña un propósito fundamental, aunque no explicitado: La misión pedagógica como apoyo indirecto a la aprobación de la ley de colonización que en aquel momento se debatía en el Parlamento (Cantera, 1968).

Para desarrollar los fines establecidos se realizarían una serie de talleres:

Misión de la Escuela y el Maestro

Ventajas de la organización legal de la familia

Derechos y deberes de los ciudadanos (expuesto por un estudiante de derecho). ${ }^{14}$

Higiene individual y colectiva y profilaxis de las enfermedades infecto-contagiosas (tratado por un estudiante de Medicina)

Agronomía: orientación científica para la mayor explotación de la tierra.

Economía doméstica, puericultura; alimentación racional; el hogar.

Educación física.

Educación estética: cantos escolares; cine; discoteca; teatro de títeres; dibujo. (Samonatti, 1946, p.256)

En el campo, los misioneros, se encontraron con la necesidad de adaptar las actividades planificadas al medio rural y al recelo del hombre de campo, no habituado "al contacto con gentes que den conferencias, ni le interesan, seguramente, los temas que en ellas se desarrollan" (Castro, 1985, p.23). Ante, las dificultades, establecen una rutina de trabajo: a la mañana salida en grupos a recorrer los ranchos invitando a las personas para la fiesta, conversar con ellas, averiguar sus modos de vida y llevarles algunas cosas (ropa y comida). Otros se quedaban en la escuela, realizando talleres y recreación. A la tarde se empezaba la función y continuaba hasta el atardecer: títeres, recitados, música, conferencias y cine.

En los intervalos servían un plato de polenta o avena caliente: "Entonces sí, para ellos, la fiesta fue completa" (Castro, 1985, p.29).

Tras el trabajo de la misión, su evaluación fue realizada en la Unión del Magisterio de Montevideo:

... El acto se inició a las 18:30 haciendo uso de la palabra el Sr. Cesar Vallejo, quien en términos generales destaca que la misión cumplida había llegado a su cometido ampliamente ya que los propósitos buscados en el sentido de irradiar una acción cultural en aquel medio, se había logrado.

Hizo presente asimismo [...] que la experiencia dejada por esta jira, permitirá abrir nuevas posibilidades para encarar en el futuro el envió [sic.] de otras misiones como la señalada.

Luego habló el señor José P. Nacimiento, quien expresó [...], que es necesario el envío continuo de nuevas misiones culturales, con el fin de llevar a dicha zona y a otras tantas más en idénticas condiciones que aquella, un material y una enseñanza social y pedagógica que permita que el nivel de vida ambiente, llegue por lo menos al límite a que tiene el derecho el habitante pobre de nuestra campaña. 
Seguidamente habló la estudiante Srta. Marta Mercader, quien puso de relieve que la jira había permitido constatar el estado de insuficiencia social de la zona visitada, por lo que es necesario que los poderes públicos se aboquen a una legislación agraria, que permita la creación de fuentes permanentes de trabajo para los que hoy son en aquella localidad permanentes desocupados.

[...] Los niños de esa zona -recalcó- se crían en la más completa orfandad y carecen de todo lo necesario, tanto en el orden físico como en el moral.

Puntualizó finalmente que es necesario una inmediata y racional intervención de los poderes públicos que, en el estudio de la legislación, sobre la reforma agraria encontrarían la solución de los graves problemas que afecta a Caraguatá. ${ }^{15}$ (“La Misión estudiantil que se trasladó a Caraguatá, informa sobre sus resultados”, 27 de julio 1945, La Mañana, p. 5)

\section{Las misiones posteriores}

La denuncia pública de la situación de miseria en que se vivía en los rancheríos permitió mantener y reproducir la experiencia. Así, entre los años 1945 y 1948, desde los Institutos Normales se planifican y organizan misiones a diferentes rancheríos del país, a razón de dos por año, durante el período de las vacaciones de los meses de julio y setiembre, de diez a quince días de duración (misiones viajeras) ${ }^{16}$ (Cantera, 1958, p.119). Estudiantes y egresados de las Facultades de Agronomía, Veterinaria, Arquitectura, Ingeniaría, Derecho, Ciencias Económicas, Medicina, Odontología, Servicio Social, fueron uniéndose a la Asociación de Estudiantes de Magisterio y participando de todas las fases de planificación, ejecución y evaluación de las Misiones Socio-Pedagógicas.

Cada año se renovaba una parte de los integrantes, de modo que los “veteranos" servían de apoyo a los recién incorporados. Algunos llegaban ingenuamente movidos por el deseo de servir. Muy pronto pasaban de la omnipotencia juvenil a la iniciación de una actitud política para el análisis del problema del rancherío. El planteamiento idealista de "ir a llevar" con que el estudiante de la ciudad partía hacia el campo, retornaba transformado en conciencia de haber recibido: en comprensión, en conocimiento, en experiencia de la vida. (Méndez, 1987, p.22)

En el año 1949, el movimiento se expande por los diferentes departamentos, fundando Centros de Misiones, encargados de la coordinación del trabajo en las misiones entre los grupos:

En algunos casos, muy al comienzo de la tarea, se fundó el Centro, en otros se fue creando con la evolución del trabajo, ya muy avanzado este. Lo que nos interesa destacar es el surgimiento de nuevos centros por todo el país y la vinculación que éstos mantienen por correspondencia en algunas oportunidades; en algunos casos observadores misioneros de un centro son invitados a concurrir a la misión que organiza otro. (Silveira, 196o, p.22)

El 29 de octubre de 1958, es sancionada en el Parlamento la ley orgánica donde se establece la gratuidad de la enseñanza, la autonomía universitaria, el cogobierno formado por docentes, estudiantes y egresados. En 1959, con la creación del Departamento de Extensión y Acción Social en la Universidad de la República, se organizan tres ensayos: uno urbano, otro suburbano y otro rural. El plan rural se estableció sobre la base de la experiencia de la Misión Permanente que el Centro de Misiones Socio-pedagógicas de Montevideo venía realizando en los rancheríos del Departamento de Flores (Pintos, Piedras, Costas de San José). El Centro de Misiones de Montevideo “quedó integrado al Departamento de Extensión Universitaria, supervisado y subvencionado por este último. Poco a poco, sin embargo, fue desdibujándose su individualidad hasta prácticamente desaparecer como tal". (Bralich, 1986, p.963)
15. "En 'Balance de la Misión Pedagógica', el maestro Julio Castro, recordará con ironía las controversias surgidas en el Congreso de Escuela Rural: ‘¡Cuántos de ellos, allí, reían de buena gana, recordando que el Congreso de Escuela Rural estuvimos cuatro o cinco días discutiendo si la escuela rural debía ser distinta de la urbana o si debían ser iguales!'” (Castro, 1985).

16. El plan de trabajo de una Misión Viajera, comprendía tres etapas o períodos: Pre-Misión, Misión y Post-Misión. Pre-Misión: Durante el cual el Centro de Misiones elegía el lugar a misionar, se capacitaba a los misioneros en las distintas tareas a realizar, se armaban los equipos, se reunía el material necesario para el trabajo de la Misión y realizar los trámites del viaje. Misión: Durante esta etapa la Misión se instalaba en el rancherío a misionar, siempre en el local de la escuela del lugar, y por medio de sus distintos equipos reunía al vecindario, le informaba de sus propósitos, realizaba el censo a la población, estudiaba los problemas sociales, culturales, económicos: “.... Trabaja junto con los lugareños desde el punto de vista de su recuperación y desarrollo espiritual; traza planes de acción para la solución de los distintos problemas del lugar, y propicia la creación de una comisión vecinal, organismo que desde el rancherío continuará esos trabajos en coordinación con la Misión después de su regreso" (Cantera, 1958, p.119). 


\section{Conclusiones}

Las Universidades Populares con la necesidad de concientizar a los obreros y campesinos, los Institutos Normales de Montevideo ante la tarea de formar a los futuros maestros y el movimiento de educadores rurales a través de la crítica y la denuncia confluyeron en el desarrollo de las Misiones Socio-Pedagógicas que aunaban su interés educativo con la transformación del campo uruguayo.

Por último, el valor de las Misiones Socio-Pedagógicas no radicó tanto por lo que obtuvieron en el medio, donde los necesarios cambios requerían otro tipo de medidas, sino por los efectos que la experiencia produjo en los propios misioneros:

Los misioneros se encontraron frente a una realidad que se expresaba por sí sola con irrebatible elocuencia. Aprendieron allí de golpe, brutal pero eficazmente, las contradicciones de nuestro mundo económico. Entre vacas y sin carne ni leche; entre ovejas y muriendo de frío en el campo y sin agua. Con la escuela próxima y no pudiendo ir a ella por falta de ropa. Aprendieron a ver que los niños van con túnica y hasta con corbata a la escuela, pese a que no tengan calzado alguno. Aprendieron a ver que hay gente que no conoce el Himno Nacional y hasta encontraron adultos que no conocían la moneda de uso corriente.

Aprendieron también que la escuela debe hacer otras cosas, antes que enseñar a leer y escribir. Y vieron que hay sociedades para las cuales la organización de la familia no existe y el casamiento no es otra cosa que un lujo.

Y como lo aprendieron mediante el tradicional y eficaz método de "la letra con sangre entra”, la experiencia fue para ellos doblemente fructífera. (Castro, 1985, p.36-37) 


\section{Referencias bibliográficas}

» AIAPE. (Marzo-abril 1939). Sección Interior de la Universidad Popular Central. Labor de la Sección Interior de la Universidad Popular.

» Bralich, J. (1986). Las misiones socio-pedagógicas en el Uruguay. Montevideo, Uruguay: Nordan - Comunidad.

"Cantera, F. (1958). Síntesis histórica de las misiones socio-pedagógicas. Superación, $1^{\underline{a}}$ Época, (35/36). Montevideo, Uruguay.

"_. (1968). Hacia los otros, historia y conclusiones de un ensayo de trabajo social con comunidades rurales. Montevideo, Uruguay.

»Castro, J. (1985). Cuadernos de Marcha, Tercera Época, año I (7). Montevideo, Uruguay.

"Clemente Batalla, I. (2008). La fundación de los Institutos Normales de Montevideo: un capítulo en la historia de la formación docente. Superación, $2^{\underline{a}}$ Época (1), p. 32. Montevideo, Uruguay.

"D‘Auria, L. (1964). Escuelas, Internatos e Institutos Normales del Uruguay, pp. 123- 124. Montevideo, Uruguay: Centro de Documentación y Divulgación Pedagógicas.

»Del Mazo, G. (comp.). (1941). La Reforma Universitaria, II tomo, pp. 86-88. La Plata, Argentina: Centro de Estudiantes de Ingeniería.

»Esteva, A. (1948). Las Misiones Pedagógicas. Anales de Instrucción Primaria, Época II, $X-X I(3,4,5)$. Montevideo, uruguay.

"Evolución (enero, 1912). Conclusiones aprobadas en las sesiones plenas del II Congreso Internacional de estudiantes americanos reunido en Buenos Aires en 1910.

"Gándaro, A. (inédito). El maestro Julio Macedo, su hacer y su tiempo.

"García Alonso, M. (2003). Necesitamos un pueblo. Genealogía de las Misiones Pedagógicas. Catálogo de la exposición Val del Omar y las Misiones Pedagógicas. Murcia-Madrid, España.

»Orticochea, M. (1941). Misión Pedagógica al San José. Superación, 1ª́ Época (32), p. 86. Montevideo, Uruguay.

»Palacios, L. (1908). Las universidades populares. Valencia, España.

»Samonatti, B. (1946). Misiones Pedagógicas. Anales de Instrucción Primaria, Época II, Tomo IX (2). Montevideo, Uruguay.

» Sanguiñedo, F. (2014). La FEUU ayer y hoy. Montevideo, Uruguay: Tradinco.

"Secretaría de Educación Pública (1928). Las Misiones culturales en 1927. Las Escuelas Normales rurales, p. 7. Ciudad de México, México: Secretaría de Educación Pública.

"Silveira, V. (1960). Las Misiones Sociopedagógicas en el Uruguay. Proyecto n.26 del Programa de Cooperación Técnica de la OEA, p. 22. Washington D.C., EE. UU.: Publicaciones del Centro Interamericano de Educación Rural.

"Soler Roca, M. (2011). El movimiento a favor de una nueva escuela rural. Montevideo, Uruguay.

"Van Aken, M. (1990). Los militantes. Historia del movimiento estudiantil uruguayo, p. 55. Montevideo, Uruguay: FCU 


\section{Bibliografía de consulta}

"AA.VV. (1933). Primer Congreso Nacional de Maestro. Organización de la Enseñanza. Estudios, Informes y Resoluciones. Montevideo, Uruguay: Central.

$»$. (1987). Dos décadas en la historia de la escuela uruguaya. Revista de la Educación del Pueblo. Montevideo, Uruguay.

»__. (s./f.). Informe sobre Escuela Primaria y algunos trabajos presentados en el Primer Congreso Nacional de Educación. Montevideo, Uruguay.

»Bruschera, J. (1945). Fines de la educación en el ambiente rural en relación con: a) La reforma agraria b) Evolución económico - político - social del país. [Trabajo presentado en congreso]. Inédita.

»Caetano, G. y Rilla, J. (1986). El joven Quijano, 1900-1933: izquierda nacional y conciencia crítica. Montevideo, Uruguay: De la Banda Oriental.

»Casona, A. (1941). Una misión pedagógica-social en Sanabria: Teatro estudiantil. Buenos Aires, Argentina: Patronato Hispano-Argentino de Cultura.

" Castro, J. (1944). La escuela rural en el Uruguay. Montevideo, Uruguay.

»__ (1945). Problemas generales de la enseñanza rural. Anales de Instrucción Primaria, Época II, Tomo VIII (2). Montevideo, Uruguay.

"Comando General del Ejército. (1978). Testimonio de una Nación Agredida. Montevideo, Uruguay.

»Cúneo, D. (comp.). (1978). Reforma Universitaria. Biblioteca Ayacucho. Caracas, Venezuela.

»Dirección De Enseñanza Primaria y Normal (1949). Congreso de maestros rurales, Piriápolis 1949. Anales de Instrucción Primaria, núm. Esp., época II, Tomo XIII $(1,2,3)$. Montevideo,

»Domingo, M. (1932). La escuela en la República. La obra de ocho meses. Madrid. Manuel Aguilar.

»Dura, H. (1933). Organización política y profesional del Magisterio. Primer Congreso Nacional de Maestro. Organización de la Enseñanza. Estudios, Informes y Resoluciones. Montevideo, Uruguay.

»Espínola, M. (1933). Organización de la escuela rural. En AA.VV. Primer Congreso Nacional de Maestros. Organización de la enseñanza. Estudios, informes y resoluciones. Montevideo, Uruguay.

»Evolución (marzo-junio de 1908). Relación Oficial del Primer Congreso Internacional de Estudiantes Americanos.

» Ferreiro, A. (1933). Organización de la escuela rural. En AA.VV., Primer Congreso Nacional de Maestros. Organización de la enseñanza. Estudios, informes y resoluciones. Montevideo, Uruguay.

»__. (1946). La enseñanza primaria en el medio rural. Montevideo, Uruguay: Consejo Nacional de Enseñanza Primaria y Normal.

» García, N. (1945). Concentración de maestros de Tacuarembó. Anales de Instrucción Primaria, Época II, Tomo VIII (2). Montevideo, Uruguay.

»Horticou, L. (1942). Sobre enseñanza normal. Enciclopedia de la Educación (Especial). Montevideo, Uruguay. 
»Leibner, G. (2011). Camaradas y compañeros. Una historia política y social de los comunistas del Uruguay. Tomo I. Montevideo, Uruguay: Trilce.

"Méndez, G. (1987). Las misiones socio-pedagógicas en el Uruguay. Revista de la Educación del Pueblo, 2a. Época (35). Montevideo, Uruguay.

»Muestra Didácticas de las Escuelas de Práctica de la Capital. (1946). Anales de Instrucción Primaria, Época II, Tomo IX (1). Montevideo, Uruguay.

"Oddone J. y París de Oddone, B. (2010). Historia de la Universidad de la República, Tomo II. Montevideo, Uruguay.

»Orticochea, M. (1943). Ciclo de Conferencias del Profesor Lorenzo Luzuriaga. Superación, $1^{a}$ Época (24). Montevideo, Uruguay.

» (1945). Reseña de las actividades realizadas en los Institutos Normales.

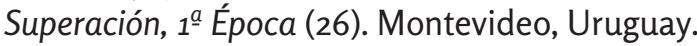

»Ortiz Benítez, L. (1952). Breve información sobre las Misiones Culturales Mexicanas. CREFAL, Ciudad de México, México.

"Pérez Montero, C. (1944). Centros de Recuperación. Anales de Instrucción Primaria, Época II, Tomo VII (3 y 4). Montevideo, Uruguay.

» Pi, R. y Wettstein, G. (1958). Rancheríos rurales y rancheríos suburbanos. En AA.VV., Rancheríos en el Uruguay. Montevideo. Oficina del Libro - CEDA.

"Samonatti de Parodi, B. (1944). Misiones Pedagógicas. Anales de Instrucción Primaria, Época II, Tomo IX, (2). Montevideo, Uruguay.

»Sanguiñedo, F. (2014). La FEUU ayer y hoy. Montevideo, Uruguay: Tradinco.

» Soler, M. (1987). El movimiento en favor de una nueva escuela rural. En AA.VV., Dos décadas en la historia de la escuela uruguaya. Montevideo, Uruguay: Revista del Pueblo.

»Sosa, J. (1937). Vida de un maestro. Buenos Aires, Argentina: Claridad.

\section{Artículos periodísticos}

" Acción (12 de marzo de 1938). Se ha creado una gran Universidad Popular Central. Se instalará en 18 de Julio y Ejido. El Día (15 de julio 1945). Una sombría realidad social fue observada por la misión pedagógica en los rancheríos de Caraguatá”, p. 7.

»El Día (12 de julio de 1945). Llego de Caraguatá la misión pedagógica escolar.

» El Día (15 de julio de 1945). Una sombría realidad social fue observada por la misión pedagógica en los rancheríos de Caraguatá.

»El país (3 de junio de 1936). Una enormidad.

» La Mañana (27 de julio 1945). La Misión estudiantil que se trasladó a Caraguatá, informa sobre sus resultados.

" Marcha (14 de julio de 1944). Se ha dado forma a una doctrina.

»Trelles, D. (31 de enero de 1941). Razón y destino de las Universidades Populares. Marcha. p. 12.

»Zavala Muniz, J. (27 de setiembre de 1940). Virtudes criollas. Marcha. 


\section{Archivos:}

» AHIINN//Fondo Institutos Normales.

» Museo Pedagógico “José Pedro Varela”.

» Biblioteca Pedagógica "Sebastian Morey Otero".

\section{Gabriel Scagliola}

Maestro, Institutos Normales de Montevideo. Magister en Memoria y Crítica de la Educación, Universidad de Alcalá de Henares y Universidad Nacional de Educación a Distancia (Madrid). Docente de Historia de la Educación en los Institutos Normales de Montevideo y en el Instituto de Formación Docente de Florida. Uruguay. Correo electrónico: gabriel.scagliola@gmail.com. 